\title{
Endovascular Treatment in Acute Ischemic Stroke: A Nationwide Survey in Korea
}

\author{
Kwon-Duk Seo, $\mathrm{MD}^{1}$, Sang Hyun Suh, $\mathrm{MD}^{2,3}$ \\ ${ }^{1}$ Department of Neurology, National Health Insurance Service Ilsan Hospital, Goyang, Korea \\ 2Department of Radiology, Gangnam Severance Hospital, Yonsei University College of Medicine, Seoul, Korea \\ ${ }^{3}$ Severance Institute of Vascular and Metabolic Research, Yonsei University College of Medicine, Seoul, Korea
}

Purpose: As endovascular therapy (EVT) has been approved as a treatment guideline for acute ischemic stroke (AIS), it has been increasing in Korea. We conducted a nationwide survey to evaluate the current status of EVT for patients with AIS in Korea.

Materials and Methods: An electronic survey was sent to a representative clinician at each hospital where EVT was available in Korea. A Google survey was used to distribute the questionnaires and receive responses from October 2017 to December 2017.

Results: Among 120 hospitals in our society, 76 participated in this survey. In $26 \%$ of the hospitals, more than 50 annual cases of EVT were performed, and 25-50 annual cases in 37\%. Fifty-six hospitals (73.7\%) achieved successful recanalization (thrombolysis in cerebral infarction $\geq 2$ b) in more than $80 \%$ of patients. Computed tomography (CT) angiography was the most common imaging modality for AIS treatment, and magnetic resonance (MR) diffusion, MR-perfusion/diffusion, and perfusion CT were frequently used in order. Non-eligibility criteria for EVT included a National Institutes of Health Stroke Scale <4 (64.5\%), the absence of MR perfusion/ diffusion mismatch (52.6\%), and a low Alberta Stroke Program Early CT Score (42.1\%). For anterior circulation stroke, $60 \%$ of Korean hospitals adopted a wider time range of " 8 hours" from symptom onset, while $70 \%$ of hospitals had a time limitation of 12-24 hours for posterior circulation stroke. The most preferred EVT device was a stentriever (89.4\%). In the failed cases due to underlying stenosis, $79 \%$ of Korean hospitals performed angioplasty or stenting for revascularization.

Conclusion: This first nationwide survey showed that most Korean hospitals conducted EVT for AIS patients according to the present guideline in the era of mechanical thrombectomy by integrating the clinical experiences of many medical institutions and specialists.

Key Words: Ischemia; Stroke

\section{INTRODUCTION}

In Korea, ischemic or hemorrhagic strokes are the third leading cause of death and the most frequent cause of disability. ${ }^{1,2}$ Since approval of tissue plasminogen activator (tPA) as the sole drug for treating acute ischemic strokes (AIS), some studies using intra-arterial thrombolytic therapy have been conducted but have failed to prove their efficacy. ${ }^{3-6}$ With the advance of technologies and devices, several randomized controlled trials of endovascular treatment (EVT) for AIS have been conducted with successful results since 2015. ${ }^{7-12}$ Based on these

\section{Correspondence to:}

Sang Hyun Suh, MD

Department of Radiology, Gangnam Severance Hospital, Yonsei University College of Medicine, 211 Eonju-ro, Gangnam-gu, Seoul 06273, Korea

Tel: +82-2-2019-4551

Fax: +82-2-3462-5472

E-mail: suhsh11@yuhs.ac

Received: July 15, 2018

Revised: August 12, 2018

Accepted: August 15, 2018
Copyright $\odot 2018$ Korean Society of Interventional Neuroradiology

This is an Open Access article distributed under the terms of the Creative Commons Attribution Non-Commercial License (http://creativecommons.org/licenses/by-nc/3.0) which permits unrestricted non-commercial use, distribution, and reproduction in any medium, provided the original work is properly cited. 
results, EVT for AIS patients has been recommended strongly as level of evidence $A^{13}$ and the Korean treatment guidelines for AlS were also revised in 2016. ${ }^{14}$

Since the introduction of neurointerventional procedures in 1994, neuroradiologists were initially involved with AIS treatment in Korea. During the past 4 years, the number of AIS patients treated with EVT has grown annually by an average of $36 \%$, and the specialized institutions for treatment of AIS and the number of specialists, including neuroradiologists, neurosurgeons, and neurologists, have also increased rapidly in Korea. ${ }^{15}$ With this nationwide survey, we tried to evaluate the present status of hospitals and clinicians related to AIS treatment in Korea, focusing on preoperative imaging diagnosis, patient selection, endovascular methods, and follow-up imaging.

\section{MATERIALS AND METHODS}

An electronic survey was sent to a representative clinician at each hospital where EVT was available in South Korea. The survey questionnaire was written in Korean and consisted of 40 single- or multiple-choice questions about general information of each hospital, preprocedural imaging evaluation, patient selection criteria, endovascular procedures, and follow-up imaging (Supplementary material). A Google Survey was used to distribute the survey questionnaires and receive responses. This survey was carried out from October to December 2017.

Questionnaire answers were evaluated with standard descriptive statistics (Microsoft Excel, Redmond, WA, USA). SPSS ver. 20.0 (SPSS Inc., Chicago, IL, USA) was used for statistical analysis. To examine the difference in the number of responses, chi-square was used for categorical variables.

\section{RESULTS}

Among 120 hospitals registered in our society, 76 participated in this survey, including 39 tertiary hospitals and 37 non-tertiary hospitals (primary and secondary hospitals).

\section{General information}

In 26\% of these hospitals (20/76), more than 50 annual cases of mechanical thrombectomy (MT) were performed, and 25-50 annual cases in 37\% (28/76). The number of tertiary hospitals that had performed more than 50 mechanical thrombectomies (MT) was 41\% (16/39), whereas only $11 \%$ $(4 / 37)$ of the non-tertiary hospitals had performed more than 50 MTs (P<0.001) (Fig. 1). In 2016, 93\% of hospitals (71/76) achieved successful recanalization (thrombolysis in cerebral infarction [TICl] 2b/3) with MT in more than $70 \%$ of AIS patients; $22 \%$ (17/76) of hospitals had recanalized successfully more than $90 \%$ of patients and 51\% (39/76) of hospitals had done so in $80-90 \%$ of patients (Fig. 2).

In participating hospitals, the distribution of clinical specialty for clinicians involved in the neurointerventional procedures was neurosurgery (54\%), radiology (30\%), and neurology (16\%). More than two clinicians managed patients with AIS in $82 \%$ of the procedures, and $91 \%$ of the hospitals ran at all hours with on-call night duty. The proportion of involvement of three or more staff in EVT was $58.8 \%$ in tertiary hospitals and $29.7 \%$ in non-tertiary hospitals $(P=0.012)$.

Thirty-eight percent (29/76) of the hospitals had a fellowship training program for EVT, in which neurosurgeons (49\%),

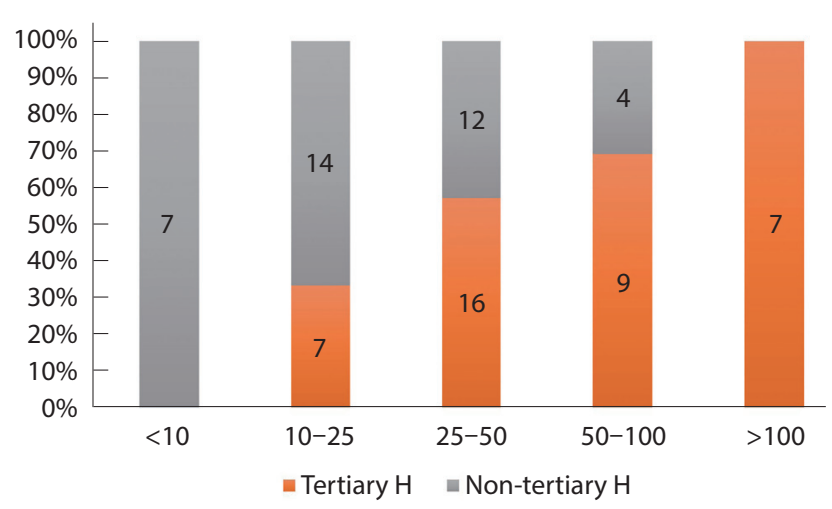

Fig. 1. Number of mechanical thrombectomy procedures per hospital in 2016 between tertiary and non-tertiary hospitals.

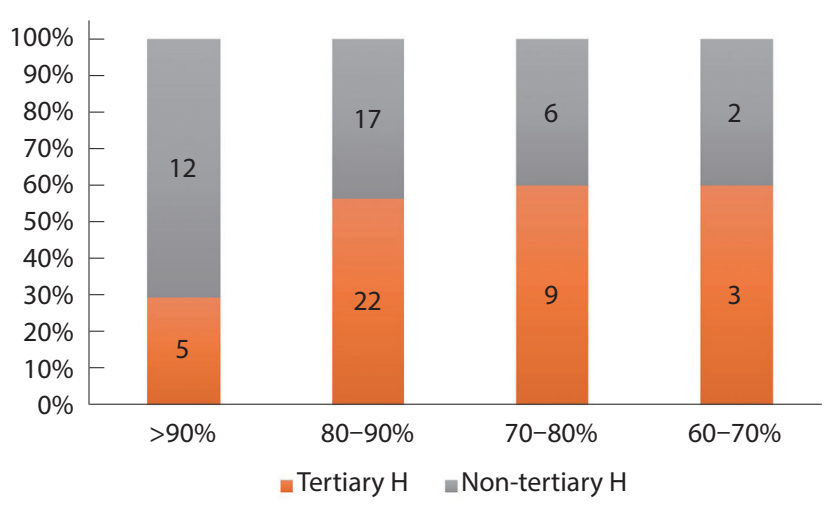

Fig. 2. Successful recanalization rate after mechanical thrombectomy at the responding hospitals. 
neurologists (28\%), and radiologists (23\%) were enrolled. Especially, the fellowship program existed in 24 tertiary hospitals. There was an inter-department consultation system in 93\% of the responding hospitals.

Specialized stroke units operated in 82\% of hospitals. More than $90 \%$ of hospitals ran biplane digital subtraction angiography, computed tomography (CT) and magnetic resonance imaging (MRI).

\section{Imaging modalities and patient selection}

$\mathrm{CT}$ angiography (CTA) was the most common imaging modality (32\%) for AIS treatment, and MR-diffusion weighted imaging (DWI, 18\%), MR-perfusion weighted imaging (PWI/ DWI, 18\%), and perfusion CT (13\%) were frequently used in that order. In case of unknown stroke onset, CTA (60\%) was the first imaging option and MR-DWI (37\%), non-contrast CT (32\%), and MR-DWI/PWI (26\%) were chosen sequentially for diagnosis.

For anterior circulation stroke, 59\% of respondents could perform EVT until 8 hours from onset of symptoms, and 25\% did within 6 hours (Fig. 3). EVT could be treated until 12 hours from symptom onset in $10.5 \%$ of hospitals. For posterior circulation stroke, EVT could be performed within 8 hours from onset in 27.6\%, 12 hours in 36.8\%, and 24 hours in 31.6\% of hospitals, respectively. In order to determine EVT for posterior circulation stroke, MR-DWI/PWI (12\%), CTA (10\%) and MRDWI (7\%) were frequently used in that order.

For the question regarding the non-eligibility for EVT, low National Institutes of Health Stroke Scale (NIHSS; <4), no DWIPWI mismatch, anatomical locations, and low Alberta Stroke Program Early CT Score (ASPECTS) were listed in that order (Table 1). For patients who were 90 years of age or older, there were some differences for treatment decisions ac-

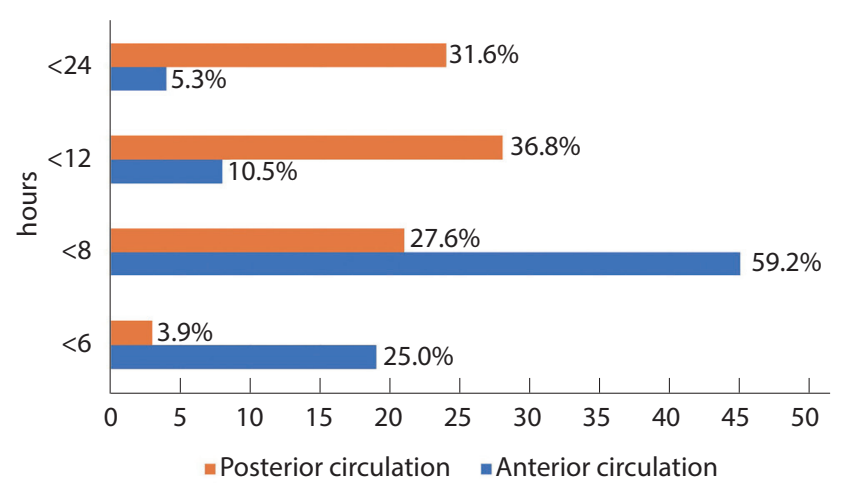

Fig. 3. Time range after symptom onset for AIS treatment between anterior and posterior circulation. AIS, acute ischemic stroke. cording to the responding hospitals, for which only $15.4 \%$ of the tertiary hospitals did not perform MT for them; whereas 48.6\% of the non-tertiary hospitals reported not performing MT. Furthermore, $10.3 \%$ of tertiary and $5.4 \%$ of non-tertiary hospitals responded that they could treat patients under any circumstances.

In patients with mild stroke symptoms (NIHSS <4), non-tertiary hospitals tended to treat more aggressively than tertiary hospitals; while only $15 \%$ of the latter treated distal middle cerebral artery (MCA) or anterior cerebral artery (ACA) occlusion, 32\% of the former would perform EVT for the same occlusion $(P<0.002)$. However, there was a similar response between tertiary and non-tertiary hospitals in case of basilar artery occlusion.

\section{Anesthetic consideration}

During EVT for AIS patients, conscious sedation was performed frequently in 56\% (41/73) of hospitals, local anesthesia in 33\% (24/73), and general anesthesia was occasionally performed in 11\% (8/73, 3 in tertiary and 5 in non-tertiary hospitals).

\section{Endovascular treatment procedure}

In total, $80 \%$ of the hospitals performed intravenous tPA followed by MT immediately, and the amount of tPA used was a full dose $(0.9 \mathrm{mg} / \mathrm{kg})$ in $78 \%$. For MT, a stentriever was frequently selected as the preferred device in $89 \%$ and an aspiration device in $11 \%$ of procedures. As a first-line device, Solitaire was chosen in 68\%, Trevo in 18\%, Penumbra in 12\%, and ERIC in $1 \%$. For the second-line option, Penumbra was the highest in 42\%, Trevo in 28\%, Solitaire in 25\%, ERIC in 4\%, and Revive SE in $1 \%$ (Table 2). The number of passes by a

Table 1. Selection criteria for patients not having mechanical thrombectomy

\begin{tabular}{lcc}
\hline Variable & Tertiary & Non-tertiary \\
\hline NIHSS $<4$ & $25(64.1)$ & $24(64.9)$ \\
Old age $(>90)$ & $6(15.4)$ & $18(48.6)$ \\
Anatomical location & $13(33.3)$ & $19(51.4)$ \\
Low ASPECTS & $18(46.2)$ & $14(37.8)$ \\
No DWI-PWI mismatch & $20(51.3)$ & $20(54.1)$ \\
None of the above & $4(10.3)$ & $2(5.4)$ \\
\hline
\end{tabular}

Values are presented as number of response (\%).

NIHSS, National Institutes of Health Stroke Scale; ASPECTS, Alberta Stroke Program Early CT Score; DWI, diffusion weighted imaging; PWI, perfusion weighted imaging. 
stentriever was 3 to 5 in 64\% and more than 5 in 36\%.

The proportion of hospitals that used balloon-guiding catheters for more than $60 \%$ of their procedures was $42 \%$, and $18 \%$ of hospitals never used balloon-guiding catheters (BGC). The proportion of hospitals that used intermediate catheters for more than $60 \%$ of procedures was $42 \%$, and $16 \%$ of hospitals did not use intermediate catheters. $63 \%$ of hospitals could use both catheters together in EVT for AIS patients.

In the case of tandem occlusion, revascularization of the proximal lesion first followed by distal recanalization was preferred in $70 \%$ of hospitals. When intracranial artery occlusion with underlying stenosis occurred, angioplasty and stenting was performed in 51\% of hospitals and only angioplasty in $28 \%$. Only intra-arterial or intravenous injection of tirofiban was administered in $18 \%$ of hospitals and bypass surgery was in $3 \%$. Finally, $61 \%$ of hospitals answered that intraarterial injection of urokinase or tirofiban was used in $<10 \%$ of procedures.

\section{Follow-up imaging}

In $69.7 \%$ of hospitals, follow-up imaging was taken within 24-48 hours after the procedure (Table 3). The proportion at which the follow-up imaging study was performed immediately or 7 days after EVT was $12 \%$ and 13\%, respectively. In order to confirm the final infarction volume, the preferred imaging study was either only MRI (49\%) or CT plus MRI (42\%).

\section{DISCUSSION}

After reporting Korean neurointerventional activities in $2013,{ }^{16}$ this study is the initial investigation into the current status of EVT in the era of MT for AIS. We found most Korean hospitals had an excellent environment for AIS patients. They have a lot of medical equipment, ranking fifth in the number

Table 2. Devices preferred for mechanical thrombectomy

\begin{tabular}{lcccccc}
\hline & \multicolumn{2}{c}{ Tertiary } & & \multicolumn{2}{c}{ Non-tertiary } \\
\cline { 2 - 3 } \cline { 5 - 6 } & 1st line & 2nd line & & 1st line & 2nd line \\
\hline Solitaire & 25 & 10 & & 27 & 9 \\
Trevo & 6 & 10 & & 8 & 11 \\
ERIC & 1 & 1 & & 0 & 2 \\
Revive & 0 & 0 & & 0 & 1 \\
Penumbra & 7 & 18 & & 2 & 14 \\
\hline
\end{tabular}

of MRIs and sixth in the number of CTs among developed countries, and have high medical accessibility due to the National Medical Insurance scheme for all citizens. ${ }^{17}$ Regarding the human resource for AIS treatment, radiologists, neurosurgeons, and neurologists have been responsible for AIS treatment in most Korean hospitals. Recently, $62 \%$ of tertiary hospitals have operated a fellowship program for training specialized experts.

According to the hospitals, there was a significant difference in the number of cerebral angiography and AIS patients who underwent MT, which may be explained by the following facts: 1) Among 43 tertiary hospitals, 11 hospitals were designated as Regional Cardio-Cerebrovascular Disease Centers by the government as well as large-scale emergency centers, where a large portion of AIS patients may be transferred by an ambulance; and 2) Most Korean hospitals are open at all hours for on-call AIS management, as found in this survey. However, non-tertiary hospitals had some difficulties managing AIS patients on-call at all hours due to a lack of medical staff and specialists who could perform MT.

In the guidelines, ${ }^{18}$ treatment for AIS patients using MT can be initiated within 6 hours after the onset of symptoms and in selected patients with AlS within 6 to 24 hours who have large vessel occlusion in the anterior circulation. In this study, it is interesting that $60 \%$ of Korean hospitals adopted the wider time range of "8 hours" from symptom onset for anterior circulation stroke, and 70\% of hospitals tried to treat posterior circulation stroke within 12-24 hours from onset. CTA was primarily applied for preoperative diagnosis and treatment decision of AIS patients at most of Korean hospitals, while MRI was the first imaging tool used for posterior

\section{Table 3. Follow-up imaging modality and time}

\begin{tabular}{lc}
\hline & Value \\
\hline Imaging modality & $32(42.1)$ \\
CT and MRI & $37(48.0)$ \\
MRI & $7(9.2)$ \\
CT & \\
Time & $9(11.8)$ \\
Immediately & $53(69.7)$ \\
$24-48$ hours & $10(13.2)$ \\
1 week & $4(5.3)$ \\
Before discharge
\end{tabular}

Values are presented as number (\%).

$\mathrm{CT}$, computed tomography; MRI, magnetic resonance imaging. 
circulation stroke.

The current guidelines ${ }^{18}$ show that patients should receive MT with a stent retriever if they meet all the following criteria: 1) a prestroke modified Rankin Score (mRS) of 0 to 1; 2) causative occlusion of the internal carotid artery or MCA segment 1 (M1); 3) age $\geq 18$ years; 4) NIHSS score of $\geq 6$; 5) ASPECTS of $\geq 6$; and 6) treatment initiated (groin puncture) within 6 hours of symptom onset. In this survey, some Korean hospitals adapted aggressive criteria for AIS treatment, including a low NIHSS (<4), low ASPECTS $(<5)$ and distal MCA or ACA occlusion, which may be considered for treatment as class Illb recommendation in some circumstances.

In Korea, the stentriever was the most preferred treatment device, while an aspiration device was chosen as a second option. Although a BGC or intermediate catheter was used frequently in AIS procedures, a BGC was not used yet in 18\% of Korean hospitals. In a recent publication, ${ }^{19}$ a BGC has been known to not only have a high success rate of recanalization but also reduce the occurrence of distal emboli and operation time, and it is expected that use of a BGC will increase further.

For failed cases due to underlying stenosis, there is a trend to perform aggressive treatments in Korea, including angioplasty or stenting. Intracranial atherosclerotic stenosis (ICAS) is one of the main causes of ischemic stroke, especially in East Asia. ${ }^{20}$ The major stroke mechanism related to the ICAS is in situ thrombo-occlusion. ${ }^{21}$ When patients with ICAS are treated using MT, endothelial damage may occur, causing immediate reocclusion and failure of recanalization. ${ }^{22}$ In recent reports, tirofiban, a glycoprotein IIb/llla inhibitor, had a positive effect as an alternative in the reocclusive cases, 22,23 and angioplasty and stenting or only angioplasty have been the last resort in these failed cases. ${ }^{24,25}$

The present study has some limitations. Since the study is based only on survey responses, it is possible to infer a recent trend for AIS treatment rather than providing exact clinical data.

\section{CONCLUSION}

This study demonstrates that most Korean hospitals conducted EVT for AIS patients according to the present guideline, which provides insight into the current status of AIS treatment according to this first nationwide survey in the era of MT. As the demand for AIS treatment increases, the academic society should prepare to cope actively with expected problems, such as education and quality control for involved hospitals and specialists and prepare for the establishment of a new guideline.

\section{SUPPLEMENTARY MATERIALS}

Supplementary materials related to this article can be found online at https://doi.org/10.5469/neuroint.2018.01053.

\section{Acknowledgments}

This study was supported by the Korean Society of Interventional Neuroradiology (KSIN) research grant 2017.

\section{REFERENCES}

1. Kim JS. Stroke becomes the 3rd important cause of death in Korea; is it a time to toast? J Stroke 2014;16:55-56

2. Hong KS, Bang OY, Kang DW, Yu KH, Bae HJ, Lee JS, et al. Stroke statistics in Korea: Part I. Epidemiology and risk factors: a report from the Korean Stroke Society and clinical research center for stroke. J Stroke 2013;15:2-20

3. National Institute of Neurological Disorders and Stroke rt-PA Stroke Study Group. Tissue plasminogen activator for acute ischemic stroke. N Engl J Med 1995;333:1581-1587

4. Furlan A, Higashida R, Wechsler L, Gent M, Rowley H, Kase C, et al. Intra-arterial prourokinase for acute ischemic stroke. The proact II study: a randomized controlled trial. Prolyse in acute cerebral thromboembolism. JAMA 1999;282:2003-2011

5. Smith WS, Sung G, Saver J, Budzik R, Duckwiler G, Liebeskind DS, et al. Mechanical thrombectomy for acute ischemic stroke: final results of the multi merci trial. Stroke 2008;39:1205-1212

6. Penumbra Pivotal Stroke Trial Investigators. The penumbra pivotal stroke trial: safety and effectiveness of a new generation of mechanical devices for clot removal in intracranial large vessel occlusive disease. Stroke 2009;40:2761-2768

7. Jovin TG, Chamorro A, Cobo E, de Miquel MA, Molina CA, Rovira $A$, et al. Thrombectomy within 8 hours after symptom onset in ischemic stroke. N Engl J Med 2015;372:2296-2306

8. Goyal M, Demchuk AM, Menon BK, Eesa M, Rempel JL, Thornton J, et al. Randomized assessment of rapid endovascular treatment of ischemic stroke. N Engl J Med 2015;372:1019-1030

9. Campbell BC, Mitchell PJ, Kleinig TJ, Dewey HM, Churilov L, Yassi 
$\mathrm{N}$, et al. Endovascular therapy for ischemic stroke with perfusion-imaging selection. N Engl J Med 2015;372:1009-1018

10. Berkhemer OA, Fransen PS, Beumer D, van den Berg LA, Lingsma HF, Yoo AJ, et al. A randomized trial of intraarterial treatment for acute ischemic stroke. N Engl J Med 2015;372:11-20

11. Nogueira RG, Jadhav AP, Haussen DC, Bonafe A, Budzik RF, Bhuva P, et al. Thrombectomy 6 to 24 hours after stroke with a mismatch between deficit and infarct. N Engl J Med 2018;378:11-21

12. Albers GW, Marks MP, Kemp S, Christensen S, Tsai JP, Ortega-Gutierrez S, et al. Thrombectomy for stroke at 6 to 16 hours with selection by perfusion imaging. N Engl J Med 2018;378:708718

13. Powers WJ, Derdeyn CP, Biller J, Coffey CS, Hoh BL, Jauch EC, et al. 2015 American Heart Association/American Stroke Association focused update of the 2013 guidelines for the early management of patients with acute ischemic stroke regarding endovascular treatment: a guideline for healthcare professionals from the American Heart Association/American Stroke Association. Stroke 2015;46:3020-3035

14. Hong KS, Ko SB, Yu KH, Jung C, Park SQ, Kim BM, et al. Update of the Korean clinical practice guidelines for endovascular recanalization therapy in patients with acute ischemic stroke. J Stroke 2016;18:102-113

15. Suh SH. The annual trends between neurointerventional and neurosurgical procedures in Korea: analysis using hira data from 2010 to 2016. Neurointervention 2017;12:77-82

16. Lee YM, Hwang SM, Kim EH, Lee DG, Shim JH, Suh DC. Current status of neurointerventional activities in Korea. Neurointervention 2013;8:65-67

17. Chun CB, Kim SY, Lee JY, Lee SY. Republic of Korea. Health system review. Health Syst Transit 2009;11:1-184

18. Powers WJ, Rabinstein AA, Ackerson T, Adeoye OM, Bambakidis
NC, Becker K, et al. 2018 guidelines for the early management of patients with acute ischemic stroke: a guideline for healthcare professionals from the American Heart Association/American Stroke Association. Stroke 2018;49:e46-e110

19. Brinjikji W, Starke RM, Murad MH, Fiorella D, Pereira VM, Goyal M, et al. Impact of balloon guide catheter on technical and clinical outcomes: a systematic review and meta-analysis. J Neurointerv Surg 2018;10:335-339

20. Holmstedt CA, Turan TN, Chimowitz MI. Atherosclerotic intracranial arterial stenosis: risk factors, diagnosis, and treatment. Lancet Neuro/ 2013;12:1106-1114

21. Kim JS, Nah HW, Park SM, Kim SK, Cho KH, Lee J, et al. Risk factors and stroke mechanisms in atherosclerotic stroke: intracranial compared with extracranial and anterior compared with posterior circulation disease. Stroke 2012;43:3313-3318

22. Kang DH, Kim YW, Hwang YH, Park SP, Kim YS, Baik SK. Instant reocclusion following mechanical thrombectomy of in situ thromboocclusion and the role of low-dose intra-arterial tirofiban. Cerebrovasc Dis 2014;37:350-355

23. Ernst M, Butscheid F, Fiehler J, Wittkugel O, Alfke K, Jansen O, et al. Glycoprotein Ilb/Illa inhibitor bridging and subsequent endovascular therapy in vertebrobasilar occlusion in 120 patients. Clin Neuroradio/ 2016;26:169-175

24. Yoon W, Kim SK, Park MS, Kim BC, Kang HK. Endovascular treatment and the outcomes of atherosclerotic intracranial stenosis in patients with hyperacute stroke. Neurosurgery 2015;76:680686; discussion 686

25. Baek JH, Kim BM, Kim DJ, Heo JH, Nam HS, Yoo J. Stenting as a rescue treatment after failure of mechanical thrombectomy for anterior circulation large artery occlusion. Stroke 2016;47:23602363 